\title{
CADENAS PRODUCTIVAS: Una alternativa para afrontar la integración regional ${ }^{*}$
}

\author{
Leslie Pierce Diez Canseco \\ GERENTE GENERAL DE ALICORP S. A. A. \\ PRESIDENTE DEL PATRONATO DE ESAN
}

\section{Resumen}

El dinamismo del proceso de integración comercial dentro de América Latina abre oportunidades y riesgos para el Perú. La gran oportunidad de acceder a mercados más grandes y más desarrollados que el nuestro significa también el riesgo de una mayor presión competitiva en el mercado interno, pues los productos de otros países ingresarán al Perú con las mismas ventajas. En este contexto, las cadenas productivas surgen como una alternativa de eficiencia colectiva, pero su desarrollo requiere de ciertas condiciones: eliminación de sobrecostos, políticas macroeconómicas coherentes, identificación de ventajas competitivas y un entorno que genere estabilidad y confianza.

nterrogado sobre si opera en un mundo

globalizado, un empresario peruano dará probablemente una respuesta afirmativa. Y es que, sin ánimos de ser reiterativo, basta comparar la evolución del PBI mundial con la evolución del comercio internacional para darnos cuenta de que cada vez más el mercado para las empresas no es su entorno local, sino el mundo ${ }^{1}$

Texto de la conferencia preparada para dictarse en ESAN el 12 de julio de 2003, durante el acto central del «Día del Graduado 2003». Cedido por el autor con anterioridad a esta fecha para publicarse en esta edición.
En numerosos debates y foros se discuten las implicancias de este escenario. Los ejecutivos están al tanto de él; pero al regresar a la cotidianeidad de sus obligaciones suelen dejar de lado este fenómeno y se concentran en la problemática que representa su mercado local. La globalización pasa a un segundo plano porque, aunque el mundo está inmerso en este

1. De acuerdo con la Cepal, en su libro Globalización y competitividad, en la década de los noventa la tasa de crecimiento promedio anual del PBI mundial fue de $3 \%$, mientras las exportaciones de bienes y servicios crecieron a $7 \%$. 
proceso, se cree que los países emergentes, salvo algunos sectores, todavía no ingresan en él. En el Perú, más bien, regresamos a la comodidad de buscar la protección de los gobiernos para cubrir ineficiencias de las cuales muchas veces ni siquiera somos conscientes.

Sin embargo, independientemente de si estamos o no preparados para enfrentarlo, este proceso se acerca rápidamente. Un análisis de lo que sucede en América Latina en cuanto a políticas de integración permite identificar una clara tendencia hacia la integración comercial dentro de cada uno de los bloques económicos existentes en el hemisferio, con la finalidad de ampliar mercados (CAN, Mercosur y Nafta). Igualmente, las relaciones entre los diferentes bloques ponen en evidencia una orientación cada vez mayor hacia la integración regional.

El Perú no es ajeno a este fenómeno regional, por no decir global, y desde mediados de la década de los noventa ha iniciado un fuerte proceso de apertura económica. Según un estudio realizado por la Universidad de Tuck, en el año 2000 el Perú ocupaba el quinto puesto en el ranking de apertura económica de los mercados emergentes, y el segundo puesto en América Latina, después de Chile.

Para ilustrar este proceso en términos prácticos, se pueden mencionar los acuerdos de integración más importantes en los que el Perú participa actualmente. En primer lugar, el Perú es miembro activo de la Comunidad Andina (CAN), donde participa con Ecuador, Colombia, Venezuela y Bolivia. Si bien nuestro país se retiró de la CAN a comienzos de los noventa, se reincorporó definitivamente en el año 1997. Dentro de la CAN, el proceso de integración ha evolucionado favorablemente y la zona de libre comercio funciona de manera satisfactoria para la mayoría de los países miembros, salvo para el Perú. Sin embargo, es sumamente importante mencionar que nuestro país ha iniciado una nueva fase de desgravación y ha anunciado que para el año 2005 todas las mercancías procedentes de la Comunidad Andina serán liberadas de aranceles. Ésta es la realidad que deberán enfrentar las empresas.

Entre las negociaciones que la CAN ha emprendido con otros bloques comerciales, para el caso peruano es de especial interés el proceso de integración con el Mercado Común del Sur (Mercosur). Actualmente, las tratativas entre ambos bloques comerciales se centran en desarrollar un tratado de libre comercio. Dentro de los objetivos planteados en las negociaciones resalta el siguiente: $« \ldots$ antes del 31 de diciembre del presente [2003] deberá estar concluida la negociación de un área de libre comercio entre ambos bloques». La posición del Perú con relación a este acuerdo es claramente favorable, más aún si se tiene en cuenta que se está impulsando una alianza estratégica con el Brasil, bajo el concepto de facilitarnos el acceso a un mercado mucho más grande (el Brasil, con más de 170 millones de habitantes), y de buscar que el país se convierta en un actor clave para agilizar el proceso de integración entre ambos bloques económicos.

Específicamente, dentro de la alianza estratégica firmada con el Brasil se ha acordado la construcción de un corredor vial que una el océano Atlántico con el océano Pacífico para incrementar el flujo comercial no sólo entre ambos países, sino también con el mundo entero. Cabe seña- 
lar que, el año pasado, mientras el Brasil exportó al Perú 436 millones de dólares, el Perú sólo logró exportar al Brasil 217 millones de dólares, casi el 50\%.

El Perú forma parte también del Área de Libre Comercio para las Américas (ALCA), que agrupa a 33 países miembros y cuyo objetivo es concretar la integración comercial de todo el hemisferio. Si bien se espera un proceso de integración todavía en el mediano plazo, los países miembros ya han acordado concluir las negociaciones para el establecimiento de una zona de libre comercio en el año 2005.

El gobierno peruano, como se ha podido observar, está tomando una actitud proactiva en el proceso de integración de los diferentes bloques económicos de la región. Además, en forma paralela muestra la misma actitud para establecer acuerdos de integración económica con otros países. Así, por ejemplo, está buscando desarrollar un acuerdo bilateral con los Estados Unidos, aprovechando el acercamiento existente a raíz del ATPDEA. Igualmente, sigue profundizando relaciones con otros países de la región, como son México y Chile.

En efecto, actualmente el gobierno del Perú está buscando firmar un acuerdo de libre comercio con México, economía considerada como la segunda mayor de la región. Aunque el acuerdo de libre comercio no se ha firmado todavía, hay en primer término un compromiso para impulsar el comercio bilateral entre ambos países. El pacto de libre comercio podría establecerse en el transcurso del próximo año.

Con respecto a Chile, el proceso de integración comercial está cada vez más avanzado. Desde hace cinco años, el Perú ha firmado un Acuerdo de Complementación Económica (ACE) con este país con el objetivo de liberalizar el comercio entre ambos países. Al respecto, conviene mencionar que recientemente se ha dado un paso muy importante en el marco de este acuerdo, pues se han logrado desgravar cerca de 2500 partidas arancelarias: «con ello, la liberación total de aranceles pasará del $44 \%$ al $81 \%{ }^{2}$, lo cual seguramente incrementará el flujo comercial entre ambos países.

El proceso de integración comercial dentro de la región es, pues, cada vez más dinámico, y el Perú participa en él de una manera muy activa. La tendencia apunta claramente a prepararse para ingresar al mercado externo, a la generación de eficiencias competitivas para mantener el mercado interno y, por supuesto, a incrementar el intercambio comercial en la región.

\section{Oportunidades y riesgos}

Pero, ¿cuáles son las principales oportunidades y riesgos asociados que se derivan de este proceso de integración? La gran oportunidad para el Perú y los empresarios peruanos es la posibilidad de acceder a mercados mucho más grandes, más desarrollados y con mayor capacidad económica, toda vez que las barreras arancelarias no impedirán el ingreso de nuestros productos.

Con la finalidad de darnos una idea de esta oportunidad de negocio que se nos

2. Alfredo Ferrero, viceministro de Integración y Negocios Internacionales, en artículo publicado en el diario El Comercio del 2 de julio del presente. 
presenta en el corto plazo, tomaré como referencia el proceso de integración más grande de la región y en el cual el Perú participará activamente: el ALCA. Actualmente, con más de 25,6 millones de habitantes y un PBI de 60 mil millones de dólares, el Perú es un mercado que se puede considerar pequeño. Teniendo en cuenta el número de habitantes, el Perú representa escasamente el $3 \%$ de la población del ALCA; y con relación al producto bruto interno, representa solamente el $0,5 \%$ del generado por el ALCA en su conjunto. La gran oportunidad de ingresar a un mercado mucho más grande es evidente y salta a la vista.

Sin embargo, en este proceso de integración hay un trade off inherente, pues así como los productos peruanos podrán ingresar a mercados extranjeros sin mayores trabas, los productos de otros países también ingresarán sin problemas al Perú. Esto provocará, de manera casi inmediata, que la presión competitiva de nuestro mercado interno se eleve. En este sentido, el principal riesgo del Perú como nación es que sus empresas no puedan afrontar esta nueva presión competitiva. Éste es el gran reto de la empresa privada en el Perú.

Al respecto, conviene analizar el nivel de competitividad que tiene el Perú en el ámbito regional actualmente, para lo cual tomamos como referencia el Global Competitiviness Report (GCR) para el periodo 2002-2003. El GCR es un estudio anual elaborado por el Foro Económico Mundial y que enfoca la posición de un país a través de dos grandes indicadores: el Índice de Competitividad para el Crecimiento (ICC), que intenta medir el potencial de crecimiento macroeconómico de un país en el mediano plazo, y el
Índice de Competitividad Microeconómica (ICM), que intenta medir la capacidad de las empresas para generar valor con los recursos disponibles actualmente.

En el estudio mencionado, al Perú le correspondió el puesto 53 (de una muestra de 80 países) en el índice de competitividad para el crecimiento, y el puesto 66 (utilizando la misma muestra) en el índice de competitividad microeconómica. Para poder darnos una mejor idea de lo que esto significa, es importante realizar un análisis comparativo con los demás países de la región.

En cuanto al índice de competitividad para el crecimiento, el Perú ha mostrado una evolución altamente favorable en los últimos años y muestra una posición mucho más sólida que el promedio de los países miembros de la Comunidad Andina, incluso de Latinoamérica. Es importante mencionar que este resultado se explica principalmente por la estabilidad macroeconómica mostrada por el país. Lo contrario sucede a nivel microeconómico, ya que el Perú muestra no sólo una posición rezagada en el ranking general, sino que en comparación con el resto de la región se encuentra detrás del promedio. Cabe señalar que este indicador refleja la situación a corto plazo del país, lo cual puede resultar preocupante si se tiene en cuenta que los procesos de integración con los demás países de la región -como he mencionado- están por culminar a la vuelta de la esquina.

Como se puede deducir, asumir el reto que significa el proceso de integración es algo complicado. Sin embargo, el propósito de esta exposición es no sólo identificar el reto, sino mostrar cómo puede ser afrontado. 


\section{Las cadenas productivas}

Una alternativa para hacer frente a este proceso de integración que ya estamos viviendo es el desarrollo de cadenas productivas. El concepto de cadenas productivas implica la concentración sectorial y/o geográfica de empresas que desempeñan las mismas actividades 0 actividades estrechamente relacionadas entre sí (tanto hacia atrás como hacia delante), con importantes y acumulativas economías externas y la posibilidad de llevar a cabo una acción conjunta en la búsqueda de eficiencia colectiva ${ }^{3}$.

La idea que subyace al concepto de cadenas productivas es que la eficiencia de la cadena es mayor a la que obtendría cada empresa en el caso de que trabajara en forma independiente; por lo tanto, genera beneficios para todas las empresas que se encuentran en la cadena. Esto se debe a las siguientes razones:

a) La concentración de empresas en una región atrae más clientes que si estas mismas empresas trabajaran de manera aislada, por lo cual el mercado se amplía.

b) «La fuerte competencia a que da lugar esta concentración de empresas induce a una mayor especialización, división del trabajo y, por ende, una mayor productividad» ${ }^{4}$.

c) La fuerte interacción entre productores, proveedores y usuarios facilita $\mathrm{e}$ induce un mayor aprendizaje produc-

3. Definición tomada de la Cepal, de su libro Apertura económica y encadenamientos productivos, pág. 33.

4. Ibid. tivo, tecnológico y de comercialización.

d) Las repetidas transacciones en proximidad con los mismos agentes económicos redundan en menores costos de transacción.

Si bien el desarrollo de cadenas productivas trae consigo una serie de externalidades positivas que le permitirían al Perú afrontar este entorno regional caracterizado por una elevada presión competitiva, debe señalarse que para que estas cadenas productivas florezcan es necesario que existan ciertas condiciones básicas que incentiven su creación.

\section{Condiciones necesarias}

Las condiciones básicas a las que se hace referencia se podrían agrupar en tres aspectos clave: en primer lugar, la eliminación de los sobrecostos existentes, junto con el establecimiento de políticas macroeconómicas coherentes; en segundo lugar, la identificación de las ventajas competitivas dentro del país; $y$, en tercer lugar, el desarrollo de un entorno propicio que genere estabilidad y confianza.

Actualmente, las empresas que operan en el país enfrentan un entorno caracterizado principalmente por: dificultades para el acceso al crédito, elevados costos logísticos y marcados sobrecostos laborales. En el Perú, el escaso financiamiento bancario que normalmente se puede obtener es de corto plazo, y comparativamente más alto que el de los demás países de la región. Esta situación, aunada a la existencia de un mercado de capitales poco desarrollado, hace que la capacidad de apalancamiento de las empresas peruanas 
sea una limitante para la inversión en proyectos de alto valor agregado. De acuerdo con un estudio elaborado por la CAF, en el 2001 uno de los principales motivos de la escasez del crédito en el país era la baja protección efectiva a los acreedores ${ }^{5}$.

En cuanto a la logística de las operaciones, los costos que enfrentan las empresas peruanas con relación al resto de la región son elevados, debido, fundamentalmente, a la escasa infraestructura básica y a la baja cobertura de servicios existentes. Al respecto, se puede mencionar que, de acuerdo con un estudio del Instituto Peruano de Economía (IPE) realizado hacia finales del año 2002, se necesitan cerca de 18200 millones de dólares para contar con una infraestructura competitiva a nivel regional. Es necesario señalar que, según el Banco Mundial, el costo de movilizar bienes en el Perú representa el $40 \%$ del costo total, mientras que el promedio para la región es el $20 \%$. La reciente ley que fija en forma imperativa un esquema de fletes mínimos, que sólo beneficia a unas empresas del sector transporte de carga, es un ejemplo justamente de lo que no debería existir en el contexto que estamos analizando.

Adicionalmente, los servicios de puertos y aeropuertos, que están concentrados en la capital, son caros e ineficientes. Sin embargo, el gobierno está buscando mejorar estos servicios a través de la venta de concesiones para la administración de puertos; razón por la cual se espera que esta situación mejore en el mediano plazo.

5. Según la CAF, el índice de protección efectiva a los acreedores coloca al Perú en los últimos lugares de la región.
Finalmente, si bien las remuneraciones en el país son comparativamente bajas con relación al resto de la región, el problema radica en los excesivos costos laborales que deben pagar las empresas que mantienen a sus empleados en el régimen formal. Obviamente, el primer problema de este esquema es que desalienta la contratación formal de mano de obra, pero existe una externalidad negativa adicional: las empresas al contratar mano de obra de manera informal no invierten en capacitarla, lo que aunado a la baja calidad de la educación escolar hace que la fuerza laboral en el Perú sea percibida como poco preparada. A propósito de este tema, es útil mencionar que de acuerdo con un estudio elaborado por el Foro Económico Mundial en el año 2000, la calidad de la enseñanza escolar en ciencias en el Perú es la más baja de la región, y que en el índice de inversión para el desarrollo del capital humano, las empresas peruanas sólo superan a sus similares de Bolivia.

Por otro lado, si bien se perciben mejoras en el Índice de Competitividad para el Crecimiento, todavía existe un sistema tributario confuso y concentrado en una escasa cantidad de contribuyentes, lo que desalienta la formalidad. Por un afán proteccionista, el sistema tributario arancelario no solamente inhibe la inversión privada, sino que además impide el desarrollo de cadenas productivas con verdadero potencial de crecimiento y generación de valor.

En el caso del trigo, por ejemplo, se ha fijado un arancel de $20 \%$ más una sobretasa de 5\% para la importación del insumo, lo que significa una protección de $25 \%$. Este elevado arancel tiene por objetivo proteger a los pequeños agricultores, 
que ven desalentado el consumo de otros productos agrícolas como el arroz y la papa. El trigo no sólo no se puede cultivar en el Perú por razones geográficas y condiciones climatológicas, sino que está probado, a través de diversas investigaciones realizadas en el país, que los derivados del trigo no sustituyen el consumo de productos nacionales como la papa y el arroz, cuyo consumo per cápita se encuentra entre los más altos del mundo. Sin embargo, lo que no resulta coherente es que en el caso de la importación de derivados del trigo, como la harina, fideos, galletas, productos todos de mayor valor agregado y que se producen en el país, se cobrará a partir del 1 de enero del próximo año solamente $16 \%$, como consecuencia del Programa de Liberación Andino al que hemos hecho referencia al inicio de esta presentación. Esto sólo incentivaría el fortalecimiento de la industria en los países socios de la CAN en desmedro de la industria nacional. Esta situación evidentemente tendrá que ser corregida.

Lo más preocupante de esta incongruencia del sistema arancelario es que ni siquiera el pequeño agricultor se vería beneficiado con la norma, porque si la demanda es cubierta por producción extranjera, él no tendría a quién venderle el trigo que sembró y estaría condenado a seguir sembrando productos con muy baja productividad $^{6}$. Señores, el Perú no es un productor de trigo y no puede serlo: tratar de autoabastecernos de trigo significaría incorporar más de 900 mil hectáreas para la producción o incrementar el rendimiento en $1150 \%$, lo que requeriría una inver-

6. De acuerdo con el Ministerio de Agricultura, mientras la rentabilidad del espárrago por hectárea asciende a 23590 soles, la rentabilidad del trigo asciende solamente a 1430 soles por hectárea. sión de 27 billones de dólares; en consecuencia, es una opción totalmente inviable. El valor agregado nacional de la cadena productiva de los derivados de trigo se calcula en 2400 millones de dólares, es decir, 12 veces el valor de las importaciones de trigo. Así, en lugar de tratar de proteger actividades productivas en las que el Perú no puede ser competitivo, se debería fomentar que la iniciativa privada se dirija a sectores donde el país puede generar cadenas productivas de valor, capaces de afrontar este entorno global. Es el momento de entender que la industria y la agricultura se complementan y que son parte de una misma cadena productiva con los beneficios que he mencionado a lo largo de la presentación. En el país ha habido experiencias exitosas, como es el caso del espárrago o el de la uva, que está tomando un impulso tremendo. Se debe dejar de interferir en el proceso de decisión de las empresas y de fomentar ineficiencias en sectores o actividades en las que el Perú no tiene capacidad de generar valor a nivel competitivo. El concepto de que un sector de la economía deba subsidiar a otros es cosa del pasado, la globalización exige encontrar eficiencias en toda la cadena productiva; la racionalidad económica de los negocios se encarga de lo demás.

El empresariado nacional se encuentra en la obligación de invertir de manera articulada para poder desarrollar cadenas productivas que sean competitivas, no sólo porque así contribuye al desarrollo de la economía nacional en su conjunto, sino por su propio beneficio, pues si no busca generar cadenas de valor competitivas u otros mecanismos alternativos para incrementar considerablemente su eficiencia, la competencia internacional lo hará desaparecer irremediablemente. 
Sin embargo, para que las empresas se decidan a invertir es necesario un clima de estabilidad y confianza, que debe convertirse en el principal objetivo del país. De acuerdo con el último estudio de la unidad de inteligencia de The Economist ${ }^{7}$, el principal riesgo que afronta el Perú es el riesgo político, el cual es explicado, básicamente, por: el bajo nivel de popularidad del Presidente, la aparente debilidad del Ejecutivo en pleno, la frágil posición del partido de gobierno en el Congreso, la clara orientación populista que está tomando el Poder Legislativo, así como por la poca visión de los partidos de oposición, que anteponen intereses políticos y partidarios a los intereses del país.

Todos estos aspectos, aunados a la falta de seguridad jurídica para el cumplimiento de contratos y a la falta de seguridad social, agravadas por el rebrote de terrorismo, provocan que la inversión se mantenga en niveles muy bajos todavía. Pero el tiempo no espera; la integración regional es ya una realidad y se le debe afrontar conjuntamente, entre el gobierno y el empresariado nacional, dentro de los roles que a cada cual le competen.

Antes de finalizar, creo que sería conveniente señalar algunos aspectos clave de esta exposición:
- Admitamos que hay un proceso de integración en marcha que debe ser conocido por el empresariado local, tanto para aprovechar las oportunidades inherentes a éste como para hacer frente a los riesgos que trae consigo.

- Actualmente, la mayoría de las empresas no están preparadas para afrontar la nueva presión competitiva que está por implantarse en nuestro mercado.

- Una alternativa para enfrentar este entorno es la integración de diferentes gremios para el eslabonamiento de cadenas productivas eficientes.

- Sin embargo, la identificación de estas cadenas productivas y el desarrollo de las mismas es labor de la iniciativa privada, que no debe ser limitada por políticas tributarias y/o macroeconómicas absurdas.

- Paralelamente, es necesario que se genere un clima de estabilidad y confianza que incentive la inversión.

- Finalmente, el gobierno debería trabajar para disminuir los sobrecostos, que afectan la competitividad de las empresas peruanas.

La tarea que tenemos por delante no es fácil. Requiere de la responsabilidad del gobierno, los partidos políticos y, por supuesto, de los empresarios.

7. Mayo 2003. 